\section{The aging heart and estrogen}

\author{
“...many now view estrogen as harmful ... we cannot take such a simplistic \\ view, rather we need to recognize that there is much we do not understand \\ about estrogen and about aging."
}

For many years conventional wisdom was that treatment of menopausal women with estrogen relieved menopausal symptoms and helped reduce heart disease. Medicine underwent a cataclysmic change 30 years ago with the advent of the controlled, double-blinded clinical trial as a standard method to test clinical questions. Several major, randomized, double-blinded clinical trials of postmenopausal hormone replacement therapy (HRT) were undertaken in the 1990s. Unexpectedly, these trials showed an increase in coronary disease, as well as the expected increase in cancer with HRT [1,2]. Further analysis of these carefully conducted trials showed that there was, on average, a 10-year delay between the onset of menopause and the institution of estrogen therapy. This finding led to the development of the timing hypothesis, which essentially proposed that the protracted delay after the onset of menopause until estrogen replacement led to significant tissue and gene expression changes, such that estrogen production was restarted in a markedly different tissue state than when estrogen production ceased [3]. A second important observation was that the increased cardiac morbidity and mortality occurred in the first year after initiation of HRT, and that there appeared to be a possible benefit of HRT in the later years of the studies and with early replacement starting in peri-menopause $[4,5]$. New investigations have now been undertaken to determine if estrogen replacement at the onset of menopausal symptoms would be beneficial. As a result of the original clinical trials, many now view estrogen as harmful. However, we cannot take such a simplistic view, rather we need to recognize that there is much we do not understand about estrogen and about aging. We need more basic research on estrogen, estrogen loss and aging, and we need an emphasis on translation of basic research to potential clinical application. We cannot elucidate important underlying molecular mechanisms if we rely only on patient studies. To improve treatment of patients, we must have a better mechanistic understanding of the changes of aging and how estrogen loss or maintenance effects these changes.
In this brief editorial, three different important cardiovascular issues, diastolic dysfunction, vascular function and changes in the heart with aging, will be touched upon. In particular new findings with the potential for translation to clinical problems will be examined.

\section{Diastolic heart failure}

Half of all heart failure is diastolic heart failure with a normal ejection fraction, but impaired relaxation [6-8]. Diastolic heart failure is a result of pathologic hypertrophy, usually occurring secondary to poorly controlled hypertension $[6,7]$. Pathologic hypertrophy is accompanied by inadequate capillary density and fibrosis. By contrast, normal or physiologic hypertrophy occurs with exercise, pregnancy and normal growth and has normal capillary density and no fibrosis. Diastolic heart failure is thought to be more common in women and the prevalence increases with aging. Thus, women developing diastolic heart failure are likely postmenopausal and not on HRT, given the drop in HRT use in response to the clinical trials discussed above. The underlying pathophysiology with impaired diastolic relaxation leading to dyspnea is an elevated left ventricular end-diastolic pressure. An increase in left ventricular enddiastolic pressure leads to increased pressure in the pulmonary vasculature, which leads to leakiness of the vessels and pulmonary congestion, once pressure exceeds a threshold. Thus, despite the presence of good contractility, these patients become dyspneic. Diastolic dysfunction accounts for half of all heart failure diagnoses, and surprisingly, the prognosis for diastolic heart failure is equally as poor as for systolic heart failure with a 5-year survival of 50\% reported by investigators at the Mayo Clinic, Rochester, MN, USA [8]. Diastolic heart failure can be far harder to manage than systolic heart failure, where an array of medications can be used to alleviate symptoms and improve survival. Treatment is primarily $\beta$-blockers and calcium channel blockers, which slow heart rate and may improve relaxation, and diuretics to reduce symptoms of breathlessness. Treatment has not changed in decades and the results are highly unsatisfactory.
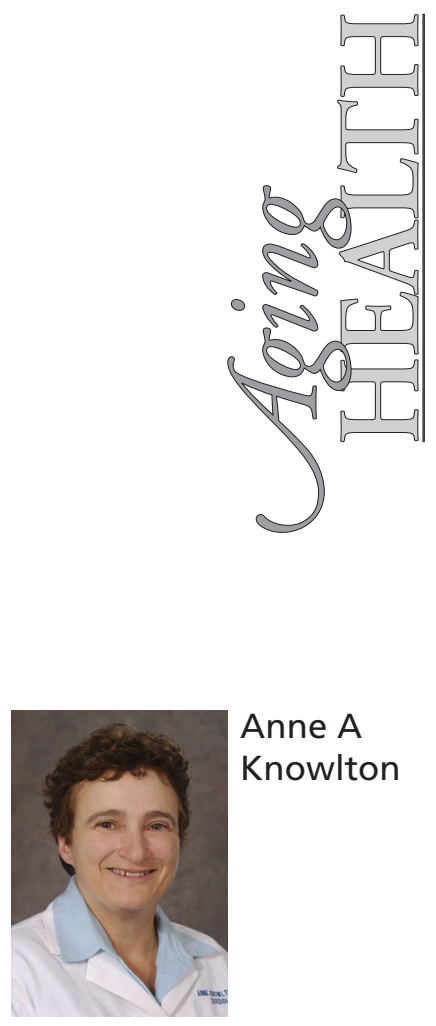

Molecular \& Cellular Cardiology, University of California, Davis, One Shields Avenue, Davis,

CA 95616, USA

Tel.: +1 5307525461

Fax: +1 5307547167

aaknowlton@ucdavis.edu

\section{Keywords}

- cardiac myocytes $\bullet$ diastolic dysfunction $\bullet$ estrogen $\bullet$ heart

- HRT • inflammation

- menopause • ROS • vascular function 
Recently, very intriguing basic investigation has been done on pathologic cardiac hypertrophy demonstrating that $17 \beta$-estradiol (E2, the most potent human estrogen) can modulate the hypertrophic response. There are three estrogen receptors: $\mathrm{ER} \alpha, \mathrm{ER} \beta$ and the most recently described GPER (also known as GPR30). E2 inhibits MMP2 expression, fibrosis and hypertrophy in pathologic hypertrophy via ER $\beta$ [9-11]. However, much of this work was done in 6-8-week-old mice, and these findings may not translate to older models or to aging humans. GPER activation can attenuate diastolic dysfunction and ventricular remodeling after ovariectomy (ovx) in a hypertensive aging rat model [12]. This work is particularly promising, as activation of GPER is not associated with some of the adverse risks of estrogen and there are selective GPER agonists available, although not yet for clinical application. Diastolic heart failure is a vexing problem and greater understanding of the underlying mechanisms is needed to develop better therapies. All of these studies were in young models, while diastolic heart failure is a disease associated with aging. As discussed below, often the response to estrogen differs between aged and adult models. Work on older models is needed to determine if these interesting affects of E2 on cardiac remodeling persist in aging models.

\section{Cardiac myocyte changes with aging}

Basic studies are important for understanding differences among adult and aged models with and without estrogen, so that we can better comprehend the mechanisms of cardiovascular changes in aging females. We have recently shown that cardiac myocytes from aged and adult (22- vs 6-month) ovx Norway Brown rats with and without immediate E2 replacement had differing responses to inducers of the protective heat shock response through activation of HSF1 (the main transcription factor activating the cardioprotective heat shock response) [13]. Neither aged group had activation of HSF1 nor increased expression of the cardioprotective protein HSP72 by simulated ischemia. Investigation into the underlying mechanism revealed that in the aged cardiac myocytes only, regardless of E2 replacement, HSF1 was inactivated by phosphorylation [13]. Furthermore, although plasma levels of IL-6 and TNF did not differ amongst the groups in this study, the aged ovx cultured cardiac myocytes had increased expression of IL-6 and TNF, suggesting that tissue levels of cytokines may be more revealing than plasma levels. This increase in IL- 6 and TNF could be improved by treatment of the cells with E2. Strikingly, the aged ovx cardiac myocytes had a markedly reduced ability to handle reactive oxygen species (ROS), the production of which is known to increase with age [14]. E2 treatment of the cardiac myocytes was unable to reduce the ROS, even though it had a positive effect on cytokine levels. Thus for the cardiac myocytes from the aged ovx rats there was a loss of the protective heat shock response, an increase in inflammatory cytokine expression and a markedly impaired ability to handle ROS with aging and loss of estrogen. E2 replacement improved many of these changes, but did not correct the inactivation of HSF1. This has important implications for patients, as ROS and inflammatory cytokines, such as TNF, are an important cause of cardiac injury.

\section{Vascular tone, nitric oxide, aging \& estrogen loss}

Estrogen has an important role in activation of endothelial nitric oxide synthase (eNOS) leading to the production of nitric oxide (NO) and vasodilation in the normal artery $[15,16]$. Employing the same model as above, vascular relaxation was found to be significantly impaired only with the combination of aging and estrogen loss, and this change was prevented by immediate E2 replacement at the time of ovx [17]. There was no difference in total NO levels amongst groups, and an NO donor did not correct relaxation. However, expression of soluble guanylyl cyclase, the receptor for $\mathrm{NO}$ in smooth muscle cells, was decreased in the aged group without E2 replacement. Relaxation could be corrected with a soluble guanylyl cyclase agonist. Hence in the aged ovx Norway Brown rat without E2 replacement, significant abnormalities were found in both the heart and vasculature of the aged ovx, which were not present in the adult ovx. Similarly, flow-mediated dilation, which is a very important physiologic response, is altered with aging and estrogen loss. Both ovx and aging attenuated flowinduced, arteriole dilation, and this was reversed in young Fischer 344 rats by E2 replacement [18]. In aged rats, flow-induced dilation was impaired in ovx and intact rats, but both had improvement with E2 replacement, likely reflecting the reduction in E2 levels in the aged rats [19]. These studies illustrate that aging and estrogen loss are both important variables that need to be studied together. Agonists of soluble guanylyl cyclase are readily available (Bayer), and could provide a new treatment for hypertension in aging, postmenopausal females. The important findings in these studies are the differences with aging 
and estrogen loss, as well as new insights into the molecular mechanisms underlying changes seen with aging and loss of estrogen. This type of research will be critical to our development of an understanding of the changes that occur with aging, which is when there is a significant increase in chronic diseases, such as heart failure, coronary disease and hypertension.

\section{The clinical trials, HRT \& increased atherosclerosis}

A key finding in the clinical trials of estrogen replacement after menopause was an increase in cardiovascular events, particularly in the first year of therapy. The mechanism of this increase was unclear, although the known increase in thrombosis associated with estrogen was considered one possible source of the increase in coronary events. Recently, we have found that late replacement of estrogen in the aging Norway Brown rat model led to increased expression of quite a few genes associated with inflammation and monocyte adhesion to the endothelium [20]. The expression of iNOS, STAT3, MADD and fibronectin all increased. TNF was only increased at the protein level, while its mRNA levels were unchanged. All of these genes are proinflammatory, and would be expected to have a negative affect on the vasculature. In addition, CD11b, a receptor for leukocyte binding to endothelial cells, was increased with late E2 replacement. Thus, changes with delayed estrogen replacement in the aged cardiovascular system resulted in an increased expression of inflammatory genes and expression of genes promoting vascular adhesion of monocytes, a critical early step in atherosclerosis.

Estrogen is a potent steroid with abundant effects on the cardiovascular system. This hormone has protective effects on cardiac myocytes and other cell types mediated through the activation of PI3K, Akt and MAP kinase pathways [21]. Estrogen also improves the lipid profile, lowering low-density lipoprotein and increasing high-density lipoprotein, accounting for some of the reduction in cardiovascular disease in

\section{References}

1. Hulley S, Grady D, Bush T et al. Randomized trial of estrogen plus progestin for secondary prevention of coronary heart disease in postmenopausal women. Heart and Estrogen/ progestin Replacement Study (HERS) Research Group. JAMA 280, 605-613 (1998).

2. Rossouw JE, Anderson GL, Prentice RL. Writing Group for the Women's Health Initiative Investigators. Risks and benefits of

premenopausal women versus age-matched men. Basic research allows investigation of underlying cell and molecular mechanisms of disease, which can lead to the development of new therapeutic approaches. However, basic research must be translated to address clinical problems. Translation of basic research to the clinic setting is a high priority for the NIH. There are plans for a National Center for Advancing Translational Science to fortify the link between basic science and clinical medicine. Clearly HRT has a complex set of effects, which include beneficial, but also deleterious ones, such as the increase in cancer (estrogen promotes cell proliferation) and thrombosis with estrogen treatment. Late estrogen replacement in the clinical trials unexpectedly led to increased cardiovascular disease. However, synthetic estrogen receptor modulators (SERMs) offer the potential of a more targeted approach. A number of SERMs have been developed and they have the potential to provide selective activation of estrogen receptors. At this time there are two SERMS, tamoxifen, primarily an estrogen receptor antagonist, and raloxifene, which has mixed agonist/antagonist properties in clinical use. In addition a sizeable number of SERMs with different properties are under development, and these raise the possibility of exploiting the protective effects of estrogen without the injurious ones to the benefit of patients. Further investigation of the underlying cellular and molecular actions of estrogen are imperative for understanding the effects of this hormone and for the development of new therapies that can harness the protective properties of estrogen.

\section{Financial \& competing interests disclosure}

AA Knowlton is supported by a Merit Award from the Veterans Administration. The author has no other relevant affiliations or financial involvement with any organization or entity with a financial interest in or financial conflict with the subject matter or materials discussed in the manuscript apart from those disclosed.

No writing assistance was utilized in the production of this manuscript.

estrogen plus progestin in healthy postmenopausal women: principal results from the women's health initiative randomized controlled trial. JAMA 288(3), 321-333 (2002).

3. Grodstein F, Clarkson T, Manson JE. Understanding the divergent data on postmenopausal hormone therapy. $N$. Engl. J. Med. 348(7), 645-650 (2003).

4. Stevenson JC, Hodis H, Pickar JH, Lobo RA. Coronary heart disease and menopause management: the swinging pendulum of HRT. Atherosclerosis 207, 336-340 (2009).

5. Grodstein F, Manson JE, Stampfer MJ. Hormone therapy and coronary heart disease: the role of time since menopause and age at hormone initiation. J. Womens Health 15(1), 35-44 (2006).

6. Ouzounian M, Lee DS, Liu PP. Diastolic heat failure: mechanisms and controversies. Nat. Clin. Pract. Cardiovasc. Med. 5(7), 375-386 (2008). 
7. Borlaug BA, Paulus WJ. Heart failure with preserved ejection fraction: pathophysiology, diagnosis, and treatment. Eur. Heart J. 32, 670-679 (2011).

8. Owan TE, Hodge DO, Herges RM, Jacobsen SJ, Roger VL, Redfield MM. Trends in prevalence and outcome of heart failure with preserved ejection fraction. N. Engl. J. Med. 355, 251-259 (2006).

9. Voloshenyuk TG, Gardner JD. Estrogen improves TIMP-MMP balance and collagen distribution inn volume-overloaded hearts of ovariectomized females. Am. J. Physiol. Regul. Integr. Comp. Physiol. 299, R683-R693 (2010).

10. Pedram A, Razandi M, O’Mahony F, Lubahn D, Levin ER. Estrogen receptor-beta prevents cardiac fibrosis. Mol. Endocrinol. 24, 2152-2165 (2010).

11. Fliegner D, Schubert C, Penkalla A et al. Female sex and estrogen receptor- $\beta$ attenuate cardiac remodeling and apoptosis in presssure overload. Am. J. Physiol. Regul. Integr. Comp. Physiol. 298, R1597-R1606 (2010).
12. Wang H, Jessup JA, Lin MS, Chagas C, Lindsey SH, Groban L. Activation of GPR30 attenuates diastolic dysfunction and left ventricle remodeling in oophorectomized mRen2.lewis rats. Cardiovas. Res. 94(1), 96-104 (2012).

13. Stice JP, Chen L, Kim SC et al. 17b-estradiol, aging, inflammation and the stress response in the female heart. Endocrinology 152, 1589-1598 (2011).

14. Podlutsky A, Ballabh P, Csiszar A. Oxidative stress and endothelial dysfunction in pulmonary arteries of aged rats. Am. J. Physiol. Heart Circ. Physiol. 298(2), H346-H351 (2010).

15. Kim KH, Moriarty K, Bender JR. Vascular cell signaling by membrane estrogen receptors. Steroids 73, 864-869 (2008).

16. Chambliss KL, Yuhanna IS, Mineo C et al. Estrogen receptor $\{$ alpha $\}$ and endothelial nitric oxide synthase are organized into a functional signaling module in caveolae. Circ. Res. 87(11), E44-E52 (2000).
17. Stice JP, Eiserich JP, Knowlton AA. Role of aging vs. the loss of estrogens in the reduction in vascular function in female rats.

Endocrinology 150, 212-219 (2009).

18. Kang LS, Reyes R, Muller-Delp JM. Aging impairs flow-induced dilation in coronary arterioles: role of $\mathrm{NO}$ and $\mathrm{H}_{2} \mathrm{O}_{2} . A m$. J. Physiol. Heart Circ. Physiol. 297, H1087-H1095 (2009).

19. LeBlanc AJ, Reyes R, Kang LS et al. Estrogen replacement restores flow-induced vasodilation in coronary arterioles of aged and ovariectomized rats. Am. J. Physiol. Regul. Integr. Comp. Physiol. 297, R1713-R1723 (2009).

20. Pechenino AS, Lin L, Mbai FN et al. Impact of aging vs. estrogen loss on cardiac gene expression: late estrogen replacement and inflammation. Physiol. Genomics 43, 1065-1073 (2011).

21. Deschamps AM, Murphy E, Sun J. Estrogen receptor activation and cardioprotection in ischemia reperfusion injury. Trends Cardiovas. Med. 20(3), 73-78 (2010). 